\title{
Hybrid Source Extraction Techniques for Periodic Signals
}

\author{
Haining Jiang ${ }^{1, a}$, Yongjian Zhao ${ }^{2, b}$ \\ ${ }^{1}$ Information Engineering Institute, Shandong University (Weihai), China \\ ${ }^{2}$ Information Engineering Institute, Shandong University (Weihai), China \\ ajian123cn@sdu.edu.cn, bzhaoyj@sdu.edu.cn
}

Keywords: Structure, Feature, Period, Mixture, Signal

\begin{abstract}
Many natural signals, such as speech signal and biomedical signal, have significant temporal structure. This work is motivated that the majority of physiological signal mixtures show some degree of periodicity and statistical nonstationarity. In fact, the nonstationarity has testified itself to be variations in period as a function of time. By exploiting the linear autocorrelation features of desired signal, a hybrid method is proposed for source signal extraction. Extensive computer simulations demonstrate the utility of the introduced techniques.
\end{abstract}

\section{Introduction}

Blind source separation (BSS) is a statistical and computational technique which aims to find underlying factors or components from multivariate statistical data. What distinguishes BSS from other methods is that it seeks for components that are both statistically independent and nongaussian. Recently, BSS technique has received much research attention due to its potential application in biomedical engineering, speech enhancement, sonar and telecommunications[1,2].

Traditional BSS techniques try to separate all source signals from mixtures simultaneously. In many practical applications such as biological signal processing, a large number of sensors are available while only one or a very few source signals are desired. For example, in EEG or MEG analysis, one obtains typically more than 64 sensor signals but only several source signals (e.g. the periodically evoked brain potentials) are desired while the rest are considered to be interfering noise $[3,4]$. Fortunately, these components are statistically independent and nongaussian. In other words, one can use BSS techniques to separate desired source signal from its mixture. In general, by traditional BSS all components are recovered from their mixture simultaneously, thus introducing heavy computational load and costing time. It is essential to develop reliable and effective source extraction methods which enable us to extract only one or a few desired source signals. How to extract specific sources of interest is called blind source extraction (BSE), which is a special branch of BSS. In contrast to traditional BSS, BSE provides more flexibility and has some potential advantages in terms of computational complexity and extraction of only the desired source signal. At present, BSE technique has become an increasingly popular data analysis technique.

Until now, a few BSE algorithms have been proposed for extraction of a specific signal by using some priori information, such as non-Gaussianity [5], smoothness or linear predictability [6,7], coding complexity [8,9], etc. For instance, Lu and Rajapakse [7] introduce a method on the basis of higher-order statistics. This method needs a priori information (called reference signal) about the period and the phase of the desired source signal. The reference signal is the one and only the one that is closest to the desired signal on the basis of a specific measure. The reference signal is of vital importance to this method. Both the shape and the phase of the reference signal may strongly affect the extraction results. Thus in some applications the reference signal is difficult to construct, at least in an automatic manner.

Many natural signals, such as speech signal and biomedical signal, have significant temporal structures[2,3]. This work is motivated by the observations that the majority of physiological signal measurements exhibit some degree of periodicity and statistical nonstationarity. In fact, the nonstationarity has testified itself to be variations in period as a function of time[4]. By exploiting the 
linear autocorrelation features of the desired signal, we present a hybrid method for source extraction which has new insights about BSE. Extensive computer simulations demonstrate the utility of the introduced techniques.

\section{Source Extraction Techniques for Periodic Signals}

Generally speaking, the basic mixture model for noise-free BSS/BSE problem can be described as follows:

$$
x(t)=A s(t)
$$

where $s(t)=\left(s_{1}(t), \cdots, s_{n}(t)\right)^{T}$ denotes the original independent sources, $x(t)=\left(x_{1}(t), \cdots, x_{n}(t)\right)^{T}$ denotes mixtures of original sources and $A$ is an $n \times n$ unknown mixing matrix.

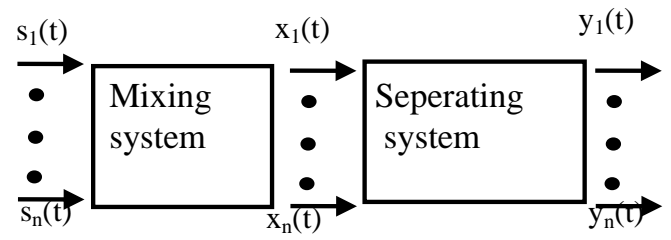

Fig.1. Block diagram of BSS problem.

Fig. 1 shows a block diagram of the traditional BSS architecture. Here source signals $s(t)$ pass through an unknown mixing system and signal mixtures $x(t)$ are obtained correspondingly. By the traditional BSS techniques, the original signals are recovered to $y(t)$ up to a scalar from their mixture simultaneously, subject to the ambiguities of permutation and scaling.

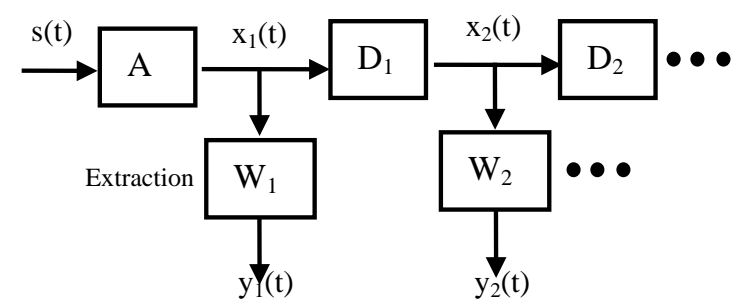

Fig.2. Block diagram of BSE and deflation.

Fig. 2 shows a general BSE architecture for extracting one or a few source singals. It consists of two principal stages: extraction and deflation. The mixtures first undergo an extraction stage to recover one source signal. If one wants to extract another source signal, the signal mixtures undergo a deflation stage and then the extracted source is removed. Subsequently, the new deflated mixtures of the remaining sources undergo the next extraction stage to recover another source signal. In contrast to traditional BSS, BSE is flexible and can extract source signals sequentially.

To formulate the BSS/BSE problem, one must introduce a reliable cost function, which is greatly dependent on the parameters of the specified neural network model in (1). Maximization or minimization of the cost function should make the output suffice the statistical conditions of stochastic independence and other inherent features. Many natural signals exhibit some degree of temporal structure. To extract a desired source signal, we introduce a single neural processing unit as follows:

$$
\begin{gathered}
y(t)=w^{T} x(t) \\
y\left(t-\tau_{k}\right)=w^{T} x\left(t_{k}\right)
\end{gathered}
$$


where $w=\left(w_{1}, \cdots w_{n}\right)^{T}$ is the weight vector, and $\tau_{k}$ is a certain time delay. We construct the following constrained maximization problem based on linear autocorrelation of desired source signals:

$$
\max _{\|w\|=1} \psi_{1}(w)=E\left\{y(t) y\left(t-\tau_{k}\right)=E\left\{\left(w^{T} x(t)\right)\left(w^{T} x\left(t-\tau_{k}\right)\right)\right\} .\right.
$$

The parameter $\tau_{k}$ can be selected as an optimal priori time delay on the basis of some information of desired source signal. As the optimal time delay cannot be acquired, the time delay can be set to one.

In many cases, one can observe several time lagged correlations for the desired source signal. Correspondigly, we modify the constrained maximization problem in (4) as

$$
\max _{\|w\|=1} \psi_{2}(w)=\sum_{k=1}^{M} E\left\{y(t) y\left(t-\tau_{k}\right)\right\}=\sum_{k=1}^{M} E\left\{\left(w^{T} x(t)\right)\left(w^{T} x\left(t-\tau_{k}\right)\right)\right\} .
$$

In deed, the constrained maximization problem in (5) can be considered as a special state of problem in (4) whereas only one time delay is avaliable.

Maximizing the cost function in (5), we exploit a flexible BSE method based on temporal correlations of source signals. The gradient of $\psi_{2}(w)$ with respect to $w$ can be acquired as follows:

$$
\frac{\partial \psi_{2}(w)}{\partial w}=\sum_{k=1}^{n} E\left\{y\left(t-\tau_{k}\right) x(t)+y(t) x\left(t-\tau_{k}\right)\right\}
$$

According to the central limit theorem and the Kuhn-Tucker conditions[1,2], one can find that at a stable point of the optimization problem in (5), the gradient of $\psi_{2}(w)$ at $w$ must point in the direction of $w$. Therefore, one can optimize the cost function in (6) by the classical fixed-point algorithm [1,2]. In other words, we can deduce

$$
\begin{gathered}
w \leftarrow \sum_{k=1}^{n} E\left\{y\left(t-\tau_{k}\right) x(t)+y(t) x\left(t-\tau_{k}\right)\right\}, \\
w \leftarrow w /\|w\| .
\end{gathered}
$$

Algorithm (7) is an improved fixed-point BSE algorithm , which is introduced by maximizing the autocorrelation of specific signal. While only one time delay is available, we can maximize the cost function in (4). Correspondingly, we can deduce

$$
\begin{gathered}
w \leftarrow E\left\{y\left(t-\tau_{k}\right) x(t)+y(t) x\left(t-\tau_{k}\right)\right\}, \\
w \leftarrow w /\|w\| .
\end{gathered}
$$

After deducing a specific vector $w$ through algorithm (7) or (8) , we can apply a demixing operation by applying vector $w$ to the mixture $x(t)$, and then $w^{T} x(t)$ is the estimated source signal. In other words, by algorithm (7) or (8), one can extract the desired source signal exclusively. If one wants to recover another source signal, he can use a deflation scheme to remove the extracted signal. The the remaining mixture experiences another extraction process through algorithm (7) or (8). As a result, another source signal can be recovered exclusively.

\section{Computer Simulations}

To check the validity and the performance of our proposed algorithm, extensive computer simulations are conducted. Due to space constraint, only one typical example is presented here. As the recordings of the fetal heart's electrical activity, fetal ECG (FECG) has progressively shown its potential for the non-invasive monitoring of the fetal health and condition $[1,3]$. However, the low amplitude FECG is always corrupted by various kinds of signals and noise, such as the maternal ECG (MECG) with extremely high amplitude, mother's respiration and stomach activity, and thermal noise from electronic equipments. Therefore, discovering a method to obtain a clear FECG has become a vital issue. The real-world ECG dataset shown in Figure 3 was distributed by De Moor [10], which was measured from a pregnant woman with eight electrodes placed at different positions on 
her body. One can see the strong and slow heart beating of the mother and the weak and fast one of the fetus. Our goal is to extract the FECG signal exclusively, which is very weak and almost only visible in s1.

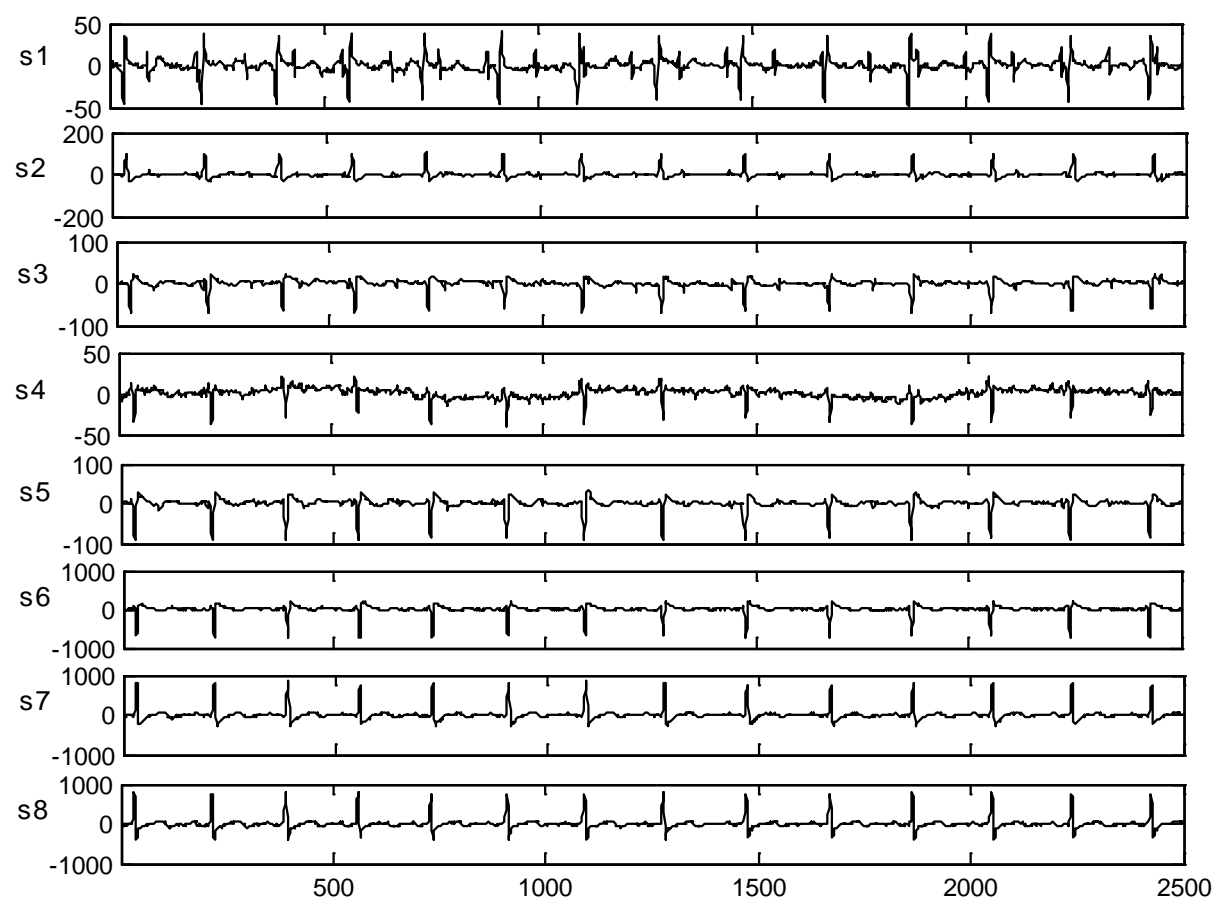

Fig. 3. The 8-channel real-world ECG data.

By carefully examining the autocorrelation of the sensor signal in s1 (where the fetal influence is clearly stronger than in the other signals) and using the fact that the fetal heart should strike every 0.5 $\mathrm{s}$ or so, we find that it has a peak at $\tau_{k}=112$ (i.e.,0.448 s). We set $\tau_{k}=112$ as the time delay for extracting the desired FECG. We ran the following BSE algorithms in turn: BCBSE [2], EVBSE [5, 8] and our algorithm(NEWBSE). The extraction results are shown in Figure 4. One can see that all these algorithms are capable of extracting the desired FCEG. However, the extraction results by the BCBSE and EVBSE algorithm are corrupted by some undesired signals or noise such as the mother's breathing artifact. In contrast, the signal extracted by the NEWBSE algorithm is clear, implying that our method can efficiently reduce the mother's breathing artifact and extract the perfect FECG.

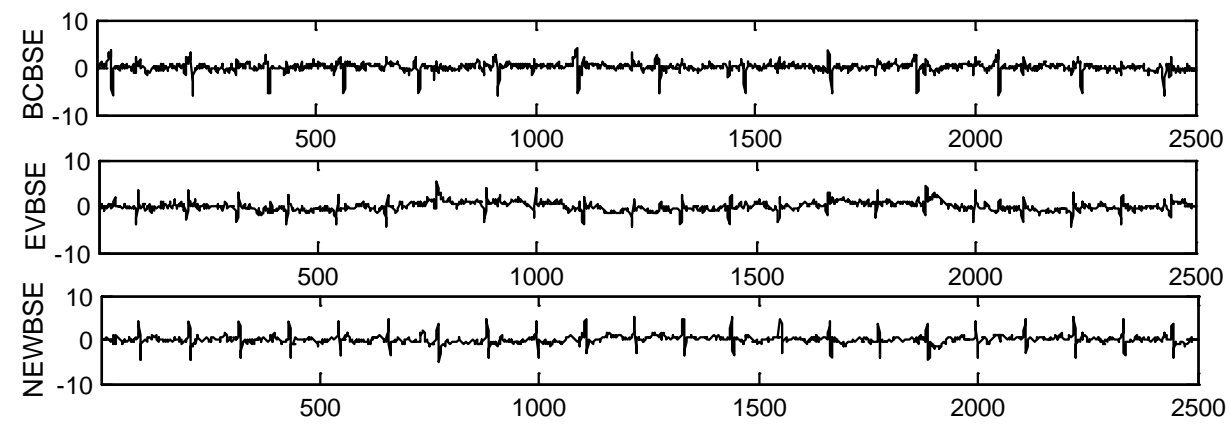

Fig. 4. Extraction results on real-world ECG data.

\section{Conclusions}

To improve signal extraction performance and expand its application, an improved BSE method is proposed by exploiting the linear autocorrelation features of desired signal. This method is very simple and do not need to choose any learning step sizes. It is adaptive and widely applicable. The traditional BSS methods aim to separate all source signals simultaneously, but they suffer from an inherent ambiguity on dilation and permutation. By the proposed method, one can choose to extract a 
desired source signal or repeat this process until the final desired one is extracted, so the source signals can be extracted in a prescribed order. In contrast to the traditional BSS techniques, the proposed method can provide us with more freedom and low computation load in separation.

\section{References}

[1] C. J. James and C. W. Hesse, Independent component analysis for biomedical signals, Physiological Measurements, vol. 26, pp. 15-39, 2005.

[2] A. K. Barros and A. Cichocki, Extraction of specific signals with temporal structure, Neural Computation, vol. 13, pp. 1995-2003, 2001.

[3] Y. J. Zhao, B. Q. Liu and S. Wang, A robust extraction algorithm for biomedical signals from noisy mixtures, Frontiers of Computer Science in China, vol. 5, no. 4, pp. 387-394, 2011.

[4] M. Liu, X. Wang and H. Zhao, Research on Illegal Information Flow in Role-based Access Control Model Based on Petri Net, ICIC Express Letters, vol.6, no.1, p139-144, 2012.

[5] Z. L. Zhang and Y. Zhang, Extraction of temporally correlated sources with its application to non-invasive fetal electrocardiogram extraction, Neurocomputing, vol. 69, pp. 894-899, 2006.

[6] T. Tsalaile, R. Sameni and S. Sanei, Sequential blind source extraction for quasi-periodic signals with time-varying period, IEEE Transactions on Biomedical Engineering, vol. 56, no. 3, pp. 646-655, 2009.

[7] W. Lu and J. C. Rajapakse, ICA with reference, Neurocomputing, vol. 69, pp. 2244-2257, 2006.

[8] Z. L. Zhang, Morphologically constrained ICA for extracting weak temporally correlated signals, Neurocomputing, vol. 71, pp. 1669-1679, 2008.

[9] R. Llinares, J. Igual, A. Salazar and A. Camacho, Semi-blind source extraction of atrial activity by combining statistics and spectral features, Digital Signal Processing, vol. 21, pp. 391-403, 2011.

[10] Information on http://www.esat.kuleuven.ac.be/sista/daisy. 